\title{
Asymmetric Line Shape of Near-field Luminescence Spectrum Induced by Stress in $\mathrm{Al}_{2} \mathrm{O}_{3}$
}

\author{
Toru Tomimatsu $^{1 *}$ and Ryo Takigawa ${ }^{2}$ \\ ${ }^{1}$ Graduate School of Science, Tohoku University, \\ 6-3 Aramaki Aza Aoba, Aobaku, Sendai 980-8578, Japan \\ ${ }^{2}$ Graduate School of Information Science and Electrical Engineering, Kyushu University, \\ 744 Motooka, Nishiku, Fukuoka 819-0395, Japan
}

(Received June 4, 2018; accepted September 5, 2018)

Keywords: near-field, stress analysis, luminescence spectroscopy, vibronic states, electron-phonon interaction

The luminescence spectroscopy of $\mathrm{Al}_{2} \mathrm{O}_{3}$ is widely used for sensing the stress in $\mathrm{Al}_{2} \mathrm{O}_{3}$ based materials. One promising approach that improves the spatial resolution of luminescence spectroscopy is the use of a near-field optical system, which is effective for assessing the localized stress in nano/microsize structural components. Here, we perform the spectral analysis of near-field excited luminescence for stressed $\mathrm{Al}_{2} \mathrm{O}_{3}$, which shows an abnormal asymmetry in line shape, which is not observed in a conventional far-field measurement. For analysis, we employ two models that include different effects. The model of stress-gradientbased spectrum broadening fails to account for the asymmetry of the luminescence spectrum. In contrast, the model including vibronic state transition yields good matching of the measured spectrum line shape, indicating that the electron-phonon coupling in $\mathrm{Al}_{2} \mathrm{O}_{3}$ affects the appearance of the asymmetric line shape. The magnitude of electron-phonon interaction as well as the effect of the excitation method on the line shape are discussed.

\section{Introduction}

Local residual stress is a key concern for hybridized structures used in nanomechanical systems or integrated circuits. Such stress localization at the joint of two materials has been widely assessed by optical spectroscopy owing to its capability of sensing stress with high spatial resolution. Among the various types of optical spectroscopy, luminescence piezospectroscopy using $\mathrm{R}$-lines is a powerful tool for stress detection in $\mathrm{Al}_{2} \mathrm{O}_{3}$ because of the strong signals and high stress sensitivity derived from the sufficient piezospectroscopic effect. ${ }^{(1-3)}$ Hence, the luminescence characteristics of $\mathrm{Al}_{2} \mathrm{O}_{3}$ have been extensively studied for various applications.

Incorporating the near-field system, which enables optical sensing beyond the diffraction limit, in luminescence spectroscopy has also paved the way to further improvement of spatial resolution up to $30-500 \mathrm{~nm} .^{(4-6)}$ This provides us a method to explore the important *Corresponding author: e-mail: tomimatu@m.tohoku.ac.jp https://doi.org/10.18494/SAM.2018.2019 
correlations between nano/micro-structures and stresses. ${ }^{(6)}$ The accuracy of stress sensing is influenced not only by measurement conditions, but also by spectral analysis. Thus, to develop the stress analysis methodology, a large amount of time for investigation is necessary. In the near-field measurement, however, no attempt at focusing on the details of the luminescence mechanism under stress has been made.

In a previous study, we investigated the effects of near-field and conventional far-field illumination on the luminescence characteristics of $\mathrm{Al}_{2} \mathrm{O}_{3} .{ }^{(4)}$ We found the apparent stressinduced asymmetry in the shape of R-lines only under the near-field illumination condition. Near-field-derived asymmetric peaks are also observed in luminescence spectra reported in other earlier works; however, the physics behind these results has not been deeply investigated. ${ }^{(7,8)}$ In this study, we perform the spectral analysis of the obtained asymmetric spectra to explore near-field luminescence origins. We introduce the effect of vibronic bands, as well as that of stress-gradient-induced broadening, in the R-line spectrum analysis.

\section{Photoluminescence Spectra under Near-field and Far-field Illuminations}

For the spectral analysis of $\mathrm{Cr}$ luminescence, we focused on the spectra obtained from stressed single-crystal $\mathrm{Al}_{2} \mathrm{O}_{3}$ with a Cr content of 0.17 mass\% (Rubicon Technology, Inc., USA). ${ }^{(4)}$ The stressed $\mathrm{Al}_{2} \mathrm{O}_{3}$ sample was prepared by thermally bonding the borosilicate glass disk (\#7740 Pyrex, Corning Glass Works, Corning, USA) to the (0001) surface of the $\mathrm{Al}_{2} \mathrm{O}_{3}$ disk at $720{ }^{\circ} \mathrm{C}$. The diameter and thickness of the disk were 7.0 and $0.5 \mathrm{~mm}$, respectively, for both the glass and $\mathrm{Al}_{2} \mathrm{O}_{3}$ layers. After bonding, the $\mathrm{Al}_{2} \mathrm{O}_{3}$ layer of the joint sample was ground to a thickness of $0.25 \mathrm{~mm}$. The surface of the $\mathrm{Al}_{2} \mathrm{O}_{3}$ layer was then polished with $1 \mu \mathrm{m}$ diamond paste to rule out the surface ruggedness and local stress induced by grinding. Because the coefficient of thermal expansion of $\mathrm{Al}_{2} \mathrm{O}_{3}$ is higher than that of glass, $\mathrm{Al}_{2} \mathrm{O}_{3}$ near the interface is subjected to in-plane tensile stresses, whereas in-plane compressive stress is applied on the $\mathrm{Al}_{2} \mathrm{O}_{3}$ top surface owing to the thermal bending during cooling. The finite element analysis (FEA) of these dissimilar materials revealed that in-plane stresses, $\sigma_{r r}$ and $\sigma_{\theta \theta}$, are homogeneous in the $r-\theta$ plane in $r<2.5$ $\mathrm{mm}$, but decay in the $z$-direction, ${ }^{(4)}$ where $z$ is the vertical axis along the sample thickness, and $r$ and $\theta$ are radial and circumferential axes from the center of the sample, respectively.

Figure 1 shows luminescence R-line spectra taken on the $\mathrm{Al}_{2} \mathrm{O}_{3}$ surface under near-field and far-field excitations. ${ }^{(4)}$ In the edge region without stress $(r \sim 3.5 \mathrm{~mm})$, both spectra for the nearfield and far-field excitations are almost similar in line frequency, line width, and intensity ratio of $\mathrm{R}_{1}$ to $\mathrm{R}_{2}$ lines. In the unstressed region, two Lorentzian functions give better matches to the measured spectra than Gaussian functions, as shown in Figs. 2(a) and 2(b). This suggests that the effects of randomly distributed strain are hindered, since the distribution of random strain is expected to give a Gaussian contribution to the line shape, whereas phonon scattering produces a Lorentzian line shape. ${ }^{(1)}$ In the region with compressive stress $(r \sim 1.0 \mathrm{~mm})$, we can see the redshift of two lines in both methods. For near-field excitation, additionally, peak broadening processes with tails in the regions below $14400 \mathrm{~cm}^{-1}$ for $\mathrm{R}_{1}$ and below $14430 \mathrm{~cm}^{-1}$ for $\mathrm{R}_{2}$ are visible [Fig. 1(a)]. Such tails, however, do not appear in the spectrum in the far-field measurement [Fig. 1(b)]. 


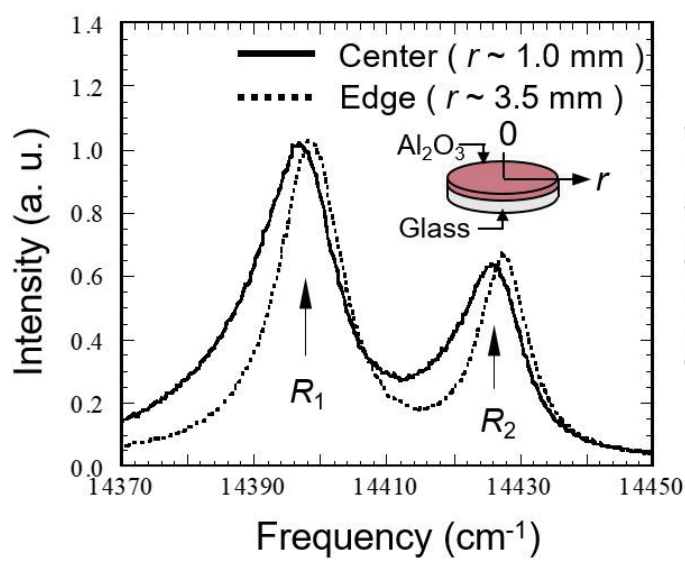

(a)

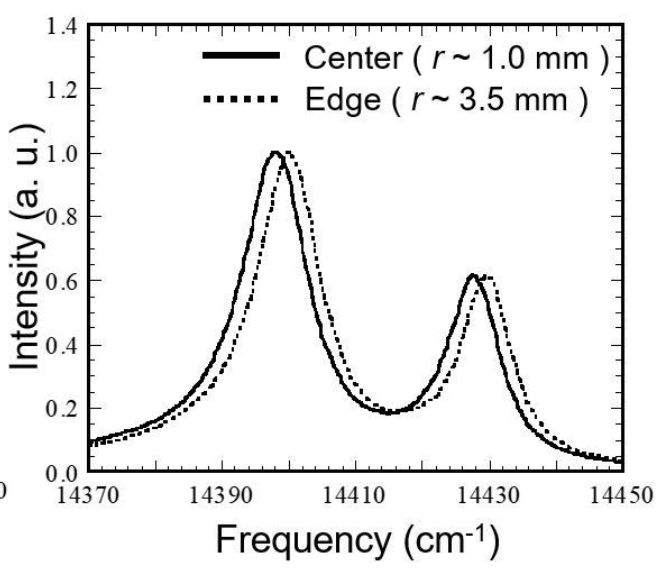

(b)

Fig. 1. (Color online) Luminescence spectra from the surface of single-crystal $\mathrm{Al}_{2} \mathrm{O}_{3}$ disk under (a) near-field and (b) far-field excitations. The $\mathrm{Al}_{2} \mathrm{O}_{3}$ (0001) surface was thermally bonded on the borosilicate glass to introduce compressible stress on the $\mathrm{Al}_{2} \mathrm{O}_{3}$ top surface, as depicted in the schematics in (a). The dimensions of the sample used in the measurement are $7.0 \mathrm{~mm}$ in diameter and 0.25 and $0.50 \mathrm{~mm}$ in thickness for the $\mathrm{Al}_{2} \mathrm{O}_{3}$ and glass layers, respectively. In the near-field measurement, the near-field probe with an aperture size of $500 \mathrm{~nm}$ was used, yielding a spatial resolution of $\sim 500 \mathrm{~nm}$. The far-field measurement with a spatial resolution of $\sim 2 \mu \mathrm{m}$ was performed using the confocal system with the objective lens (magnification: 100, numerical aperture: 0.95). ${ }^{(4)}$

(a)

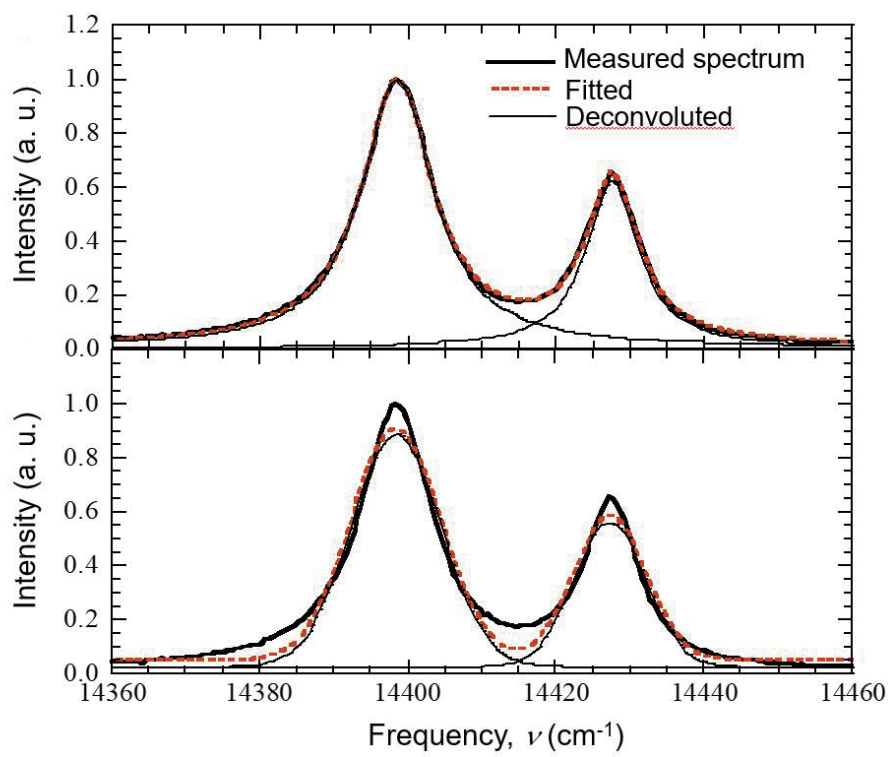

Fig. 2. (Color online) Deconvolution by fitting (a) Lorentzian and (b) Gaussian functions to $\mathrm{R}_{1}$ and $\mathrm{R}_{2}$ lines of stress-free $\mathrm{Al}_{2} \mathrm{O}_{3}$ obtained by near-field measurement in the sample edge region. 


\section{Spectral Analysis of Near-field Luminescence for Stressed $\mathrm{Al}_{2} \mathrm{O}_{3}$}

In conventional stress analysis using luminescence, it is assumed that, with homogeneous stress in $\mathrm{Al}_{2} \mathrm{O}_{3}$, the luminescence line shape is unchanged and the stress in the crystallographic coordinate system $\sigma_{i j}$ simply leads to a translational shift of the line frequency $\Delta v$ in accordance with the following piezospectroscopic relationship: ${ }^{(2)}$

$$
\Delta v=\Pi_{i j} \sigma_{i j}
$$

where $\Pi_{i j}$ is a piezospectroscopic coefficient. The translational spectral shift is clearly seen in the result for far-field excitation [Fig. 1(b)]. One of the candidates responsible for spectral distortion appearing in the near-field measurement is spatial stress inhomogeneity. Here, we investigate the stress gradient in the depth direction within the near-field illumination area and its contribution to the spectrum line shape. The effect of stress on luminescence intensity is evaluated by assuming that luminescence sensitivity is proportional to the near-field intensity profile because of the dipole-dipole interaction. By taking into account that the intensity depends on the inverse cube of the dipole-dipole distance, ${ }^{(5)}$ the near-field intensity on the $r-\theta$ plane at the depth $z$ is given by

$$
I_{N F}(z) \propto \int_{0}^{R_{\infty}} \frac{r}{\left\{r^{2}+\left(z+\frac{a}{2}\right)^{2}\right\}^{1.5}} d r
$$

where $R_{\infty}$ is the integration range and $a$ is the aperture size of the near-field probe ( $a=500 \mathrm{~nm}$ ). We set $R_{\infty}$ to $30 a$, which is the optimum value for luminescence analysis. ${ }^{(5)}$ Figures 3(a) and 3(b) show $z$ profiles of near-field intensity decay derived from Eq. (2) and in-plane stress obtained by FEA, ${ }^{(4)}$ respectively. At $z=2 \mu \mathrm{m}$, the intensity decreases to $\sim 10 \%$ of that at $z=$ $0 \mu \mathrm{m}$ and the compressive stress decreases by $\sim 7 \mathrm{MPa}$ from the value at the surface. Figure 3(c) shows the correlation between near-field intensity [Fig. 3(a)] and stress [Fig. 3(b)] at the same $z$. The conversion from the stress in Fig. 3(c) to the peak frequency shift gives us the weighting function $G(v)$, which represents the contribution of the stress gradient to the luminescence spectra. For the conversion from the stress to the peak shift, the piezospectroscopic relationship under the biaxial stress condition $\left(\sigma=\sigma_{r r}=\sigma_{\theta \theta}\right)$ is employed:

$$
\Delta v=\frac{2}{3} \Pi_{i i} \sigma
$$

where the coefficient $\Pi_{i i}$ for the $\mathrm{R}_{2}$ line is $\Pi_{i i}=7.61 \mathrm{GPa}^{-1} \mathrm{~cm}^{-1}$. (1) The stress-gradient-based line shape $I_{s g}(v)$ is then derived by convoluting the luminescence spectrum for the stress-free condition $I_{0}(v)$ with $G(v)$ as expressed by 

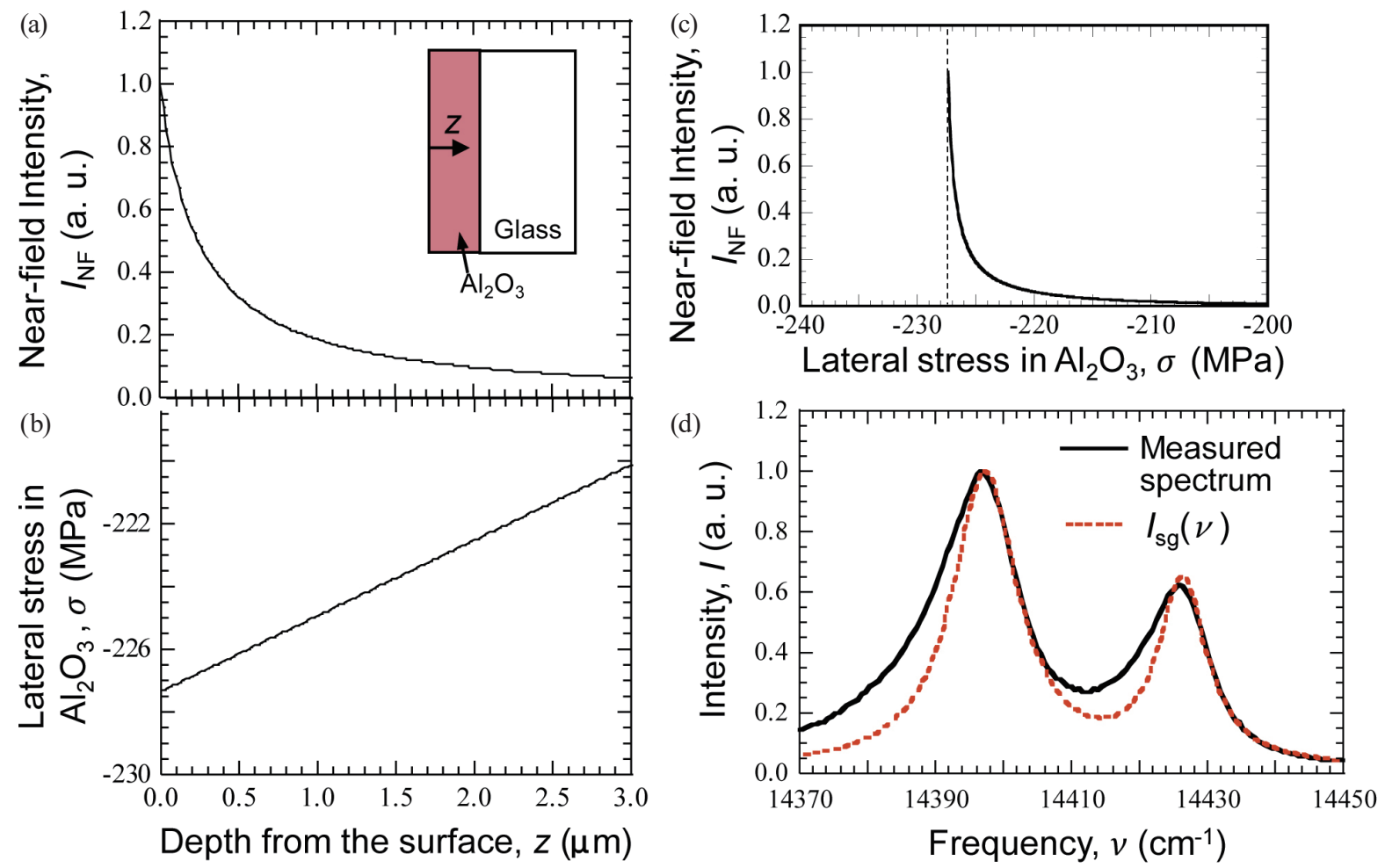

Fig. 3. (Color online) (a) Calculated near-field intensity integrated over $r-\theta$ plane at depth $z$ from the $\mathrm{Al}_{2} \mathrm{O}_{3}$ surface of the sample and (b) the lateral stress profile derived by finite element analysis, $\sigma$. (c) Relationship between $I_{N F}$ and $\sigma$ at identical $z$ plane. (d) Near-field luminescence spectrum of stressed $\mathrm{Al}_{2} \mathrm{O}_{3}$ and the calculated line shape including stress gradient effect, $I_{s g}(v)$.

$$
I_{s g}(v)=\int_{-\infty}^{\infty} I_{0}\left(v^{\prime}\right) G\left(v-v^{\prime}\right) d v^{\prime} .
$$

Figure 3(d) shows the measured spectrum under the stress condition and calculated $I_{s g}(v)$. Maximum peak frequencies of both $\mathrm{R}_{1}$ and $\mathrm{R}_{2}$ lines of $I_{s g}(v)$ are almost consistent with those of the measured spectrum; the deviations of the measured and calculated frequencies at the maximum peak intensity are less than $0.5 \mathrm{~cm}^{-1}$ for both R-lines. This indicates that our FEA and piezospectroscopic analysis well reproduce peak positions of the measured luminescence. Although $I_{s g}(v)$ agrees with the measured spectra on the higher frequency side of $\mathrm{R}_{1}$ and $\mathrm{R}_{2}$ lines, the intensity tail on the lower frequency side remains uncharacterized. The asymmetric spectrum of near-field luminescence was also observed in earlier works and discussed from the viewpoint of stress inhomogeneity. ${ }^{(7)}$ However, as evident in our spectral analysis based on the stress gradient, the asymmetry of the spectra cannot be explained by only the inhomogeneity of the spatial stress distribution.

One probable explanation for such asymmetry is the appearance of the effect of vibronic states owing to the enhanced electron-phonon interaction in the $\mathrm{Al}_{2} \mathrm{O}_{3}$ layer. R-lines observed 
in Cr-doped $\mathrm{Al}_{2} \mathrm{O}_{3}$ correspond to the energy state transition of ${ }^{2} \mathrm{E} \rightarrow{ }^{4} \mathrm{~A}_{2}$, which is affected by the interaction of $\mathrm{Cr}^{3+}$ ions and the lattice vibrations in ionic crystal. ${ }^{(9)} \mathrm{Next}$, we perform the analysis by employing the simplified line shape model including the vibronic band effect, ${ }^{(10-12)}$ which assumes the Frank-Condon optical transition, given by

$$
I_{v i b}(v) \propto \sum_{m} \exp (-S) \frac{S^{m}}{m !} B_{m}(v),
$$

where $m$ is the number of vibrational quanta, $S$ is the Huang-Rhys parameter representing the strength of electron-phonon coupling, and $B_{m}(v)$ is the line shape for an $m$-phonon process. For $m=0, B_{0}(v)$ is the zero-phonon line-shape function. We use the deconvoluted Lorentzian line shape for $B_{0}(v)$, since the luminescence spectrum under the basal stress-free condition is well fitted by the Lorentzian function in our sample [Fig. 2(a)]. $B_{m}(v)$ values for higher-order processes are obtained by $m$-times convolution with the phonon spectrum, which is assumed to be the single Gaussian function: ${ }^{(11)}$

$$
B_{m}(v) \propto \int_{0}^{\infty} B_{m-1}\left(v^{\prime}\right) \exp \left\{-\frac{\left(v-v^{\prime}-v_{0}+m v_{p h}\right)^{2}}{2 w^{2}}\right\} d v^{\prime}
$$

where $v_{0}$ and $v_{p h}$ are the frequencies corresponding to the energy of the zero-phonon line and the mean phonon energy, respectively, and $w$ is the width of the emitted phonon spectrum.

The best-fit result of Eq. (5), i.e., $I_{v i b}(v)$, to the measured spectrum is shown in Fig. 4. The optimum $S$ parameter is $S=0.6$ for stressed $\mathrm{Al}_{2} \mathrm{O}_{3}$. The problem of poor matching of $I_{s g}(v)$ in the lower frequency region of R-lines [Fig. 3(d)] is clearly resolved by using $I_{v i b}(v)$. The $S$ value for the obtained result $(S=0.6)$ is smaller than that for $\mathrm{Al}_{2} \mathrm{O}_{3}$ nanopowder, ${ }^{(12)}$ since the number of defects and the degree of disorder are considerably suppressed in bulk single-crystal $\mathrm{Al}_{2} \mathrm{O}_{3}$ compared with $\mathrm{Al}_{2} \mathrm{O}_{3}$ nanopowder.

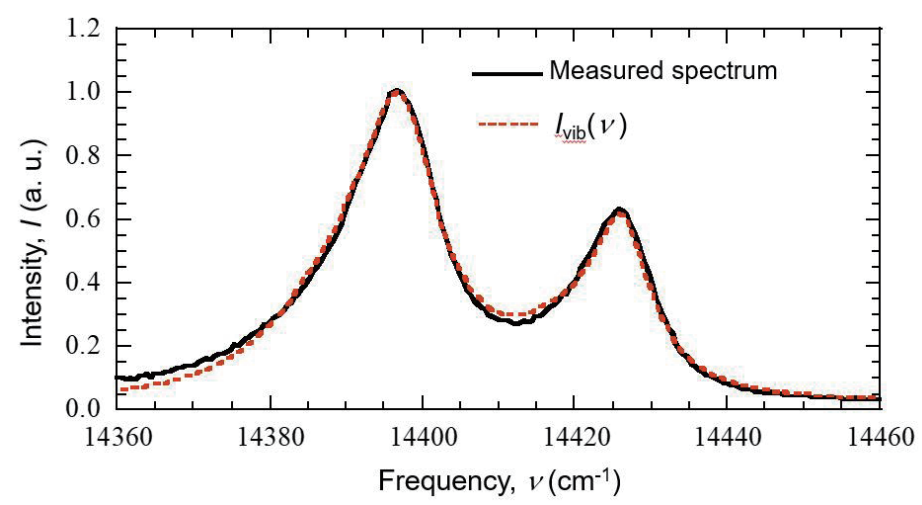

Fig. 4. (Color online) Near-field luminescence spectrum of stressed $\mathrm{Al}_{2} \mathrm{O}_{3}$ and the curves fitted using the equation based on the vibronic state effect, $I_{v i b}(v)$. 
Although the description of the vibronic states in Eq. (5) does not include the static stress effect, it is reasonable to assume that all states shift with stress in accordance with the piezospectroscopic relationship. In $I_{v i b}(v)$, the $\mathrm{R}_{2}$ basal line frequency for stressed $\mathrm{Al}_{2} \mathrm{O}_{3}$ is estimated to be $v=14426.3 \mathrm{~cm}^{-1}$, which is almost identical to that for $I_{s g}(v)$. This agreement in the peak positions between the two models supports that the fitting analysis using the vibronic state model accurately represents the physics of R-line luminescence.

The spectral analysis using Eq. (5) has a limitation because the equally gapped phonon levels are assumed by the harmonic approximation of electronic states, which is not always the case in a real vibrational band system. Accurate studies of vibronic energy levels would clarify the deviation of the measured and calculated line shapes, which is notable in the lowfrequency region and enables a more advanced analysis of the mixed effects of vibronic bands and inhomogeneous stresses.

Our measurement results also suggest the importance of the excitation field to the spectra for the stressed sample, as shown in Fig. 1. The difference in the capability to detect the local stress gradient between near-field and far-field excitations can be ruled out as the reason for the difference in line shape because stress homogeneity is maintained in the region of $r<2.5 \mathrm{~mm}$ on the surface of the sample. Thus, the in-plane spatial resolutions of two methods do not affect the detected stress. In contrast, for the depth $(z)$ direction, the steep gradient of in-plane compressive stress is produced in the $\mathrm{Al}_{2} \mathrm{O}_{3}$ layer [Fig. 3(b)]. Such a stress gradient effect, however, was not manifested as the broadening of the line shape in the far-field measurement [Fig. 1(b)], despite the fact that it excites and detects luminescence in regions deeper than those in the near-field measurement. This fact again confirms that the stress gradient has a poor contribution to the R-line shape in our sample. The important difference between the two methods that may crucially contribute to the line shape is the electric field direction of excitation or the detection efficiency. Under the far-field illumination, the wave vector component perpendicular to the sample surface is dominant, whereas the parallel wave vector component is enhanced under the near-field illumination. ${ }^{(13)}$ We speculate that the stress anisotropy of the sample may induce the anisotropic electron-phonon interaction that has a preferred direction of the electric field for generating the vibronic band effect. Future investigation regarding the effects of the stress anisotropy and electric field polarization on the luminescence will help elucidate the details of the luminescence mechanism. Our finding provides important insight into the near-field-based piezospectroscopy and its application to the stress generation area of structural components, such as the bonded interface of dissimilar materials. ${ }^{(14-16)}$

\section{Conclusions}

We studied the stress-induced asymmetry of the luminescence spectrum generated by near-field illumination. To date, such spectrum distortion in the near-field measurement has been studied from the viewpoint of stress inhomogeneity. In this study, for the first time, we performed the R-line spectral analysis including the effects of vibronic state transitions. The vibronic-state-based model is well fitted to the luminescence spectrum as compared with the stress gradient model. The estimated value of the Huang-Rhys parameter $S$ by the vibronic- 
state-based model is small $(S=0.6)$, indicating only slight electron-phonon coupling in stressed $\mathrm{Al}_{2} \mathrm{O}_{3}$. This also suggests that the appearance of an asymmetric line shape is sensitive to even a small degree of electron-phonon coupling. A study of the effects of near-field polarization and stress anisotropy on the spectrum would deepen our understanding of near-field luminescence.

\section{Acknowledgments}

This work was supported by the JSPS (KAKENHI 18K04874).

\section{References}

1 J. He and D. R. Clarke: J. Am. Ceram. Soc. 78 (1995) 1347. https://doi.org/10.1111/j.1151-2916.1995.tb08493.x

2 X. Peng and D. R. Clarke: J. Am. Ceram. Soc. 83 (2000) 1165. https://doi.org/10.1111/j.1151-2916.2000.tb01349. $\mathrm{X}$

3 J. A. Nychka and D. R. Clarke: Surf. Coat. Technol. 146 (2001) 110. https://doi.org/10.1016/S02578972(01)01455-4

4 T. Tomimatsu: Ph.D thesis, University of Tokyo, Japan (2006) (in Japanese).

5 T. Tomimatsu, Y. Kagawa, and D. R. Clarke: J. Appl. Phys. 101 (2007) 123527. https://doi. org/10.1063/1.2749465

6 T. Tomimatsu and R. Takigawa: Jpn. J. Appl. Phys. 57 (2018) 06HF01. https://doi.org/10.7567/JJAP.57.06HF01

7 M. A. Paesler, P. J. Moyer, C. L. Jahncke, C. E. Johnson, R. C. Reddick, R. J. Warmack, and T. L. Ferrell: Phys. Rev. B 42 (1990) 6750. https://doi.org/10.1103/PhysRevB.42.6750

8 C. C. Lai, P. Yeh, S. C. Wang, D. Y. Jheng, C. N. Tsai, and S. L. Huang: J. Phys. Chem. C 116 (2012) 26052. https://doi.org/10.1021/jp309024g

9 H. C. Seat and J. H. Sharp: IEEE Trans. Inst. Meas. 53 (2004) 140. https://doi.org/10.1109/TIM.2003.822010

10 B. Henderson and G. F. Imbusch: Optical Spectroscopy of Inorganic Solids: Monographs on the Physics and Chemistry of Materials (Clarendon Press, Oxford, 1989) p. 196.

11 M. Yamaga, B. Henderson, and K. P. O’Donnell: Phys. Rev. B 46 (1992) 3273. https://doi.org/10.1103/ PhysRevB.46.3273

12 S. V. Bulyarskii, A. E. Kozhevin, S. N. Mikov, and V. V. Prikhodko: Phys. Status Solidi A 180 (2000) 555. https://doi.org/10.1002/1521-396X(200008)180:2<555::AID-PSSA555>3.0.CO;2-D

13 B. Hecht, B. Sick, U. P. Wild, V. Deckert, R. Zenobi. O. J. F. Martin, and D. W. Pohl: J. Chem. Phys. 112 (2000) 7761. https://doi.org/10.1063/1.481382

14 J. H. Han, M. Takenaka, and S. Takagi: Jpn. J. Appl. Phys. 55 (2016) 04EC06. https://doi.org/10.7567/ JJAP.55.04EC06

15 R. Takigawa, E. Higurashi, T. Suga, and T. Kawanishi: Sens. Actuators, A 264 (2017) 274. https://doi. org/10.1016/j.sna.2017.08.015

16 R. Takigawa, E. Higurashi, and T. Asano: Jpn. J. Appl. Phys. 57 (2018) 06HJ12. https://doi.org/10.7567/ JJAP.57.06HJ12 